\title{
A Quenched Oscillator Network for Pattern Formation in Gene Expression
}

\author{
Justin Hsia, William A. Holtz, Daniel C. Huang, Murat Arcak, Michel M. Maharbiz \\ Department of Electrical Engineering and Computer Science, \\ University of California, Berkeley, 94720
}

\begin{abstract}
Biologists have long searched for reaction networks that can spontaneously generate spatial patterns in the presence of diffusion. The studies so far closely follow Alan Turing's "activator-inhibitor" model, where diffusion destabilizes a spatially homogeneous steady state. In this paper, we propose a new type of Turing system based on an oscillator that destabilizes a spatially homogeneous steady state towards a spatio-temporal pattern. We develop a model based on current experimentally-viable systems and study its benefits and limitations.
\end{abstract}

\section{INTRODUCTION}

Genetic networks which enable communication and coordination of behavior among cells in an ensemble have held the attention of developmental biologists [1], [2], [3] and theoreticians [4], [5], [6], [7] for over half a century. In particular, a vast body of literature - both theoretical [1-6],[8] and experimental [9], [10], [11] - exists which focuses on the production of patterns in gene expression, a phenomenon central to the development of multi-cellular organisms. A particularly interesting mechanism for pattern formation is diffusion-driven instability, originally proposed by Turing [12].

More recently, attempts have been made to build synthetic gene networks which generate patterns, usually via the external manipulation of diffusible substances that modulate gene expression [13], [14], [15], [16], [17]. To date, there have been no experimental demonstrations of a robust, tunable system which can break symmetry and spontaneously generate predictable gene expression patterns from an initially homogenous condition as in the Turing mechanism.

In the synthetic biology community, most efforts to achieve spontaneous generation of spatial patterns have centered around networks similar to the one originally proposed by Turing [12]: Two diffusible species (usually termed an activator and an inhibitor) interact with each other via chemical reactions that produce positive and negative interactions as in Figure 1. For an appropriate range of kinetic parameters and diffusion constants, these topologies produce spatial or spatio-temporal patterns from a homogenous initial condition perturbed by the chemical noise present in normal experimental conditions. However, this type of architecture has proven very difficult to implement using genetic networks because: (a) Turing instability requires that the steady-state occur in the linear regime of the activator-inhibitor interactions away from saturation and severely restricts the parameter range to meet the instability criteria; (b) when using systems with two diffusible components, either the diffusion constants [12] or the uptake rates [4] must be sufficiently different to allow unstable spatial modes, and significant differences are difficult to engineer; and (c) the intermediate protein steps ignored in a two-state activator-inhibitor model further restrict the parameters, possibly leaving them outside the tunable range.

Although the activator-inhibitor model is the canonical example for Turing instability, many other possible network structures exist. Indeed, as we make explicit in this paper, the essential structural property for the Turing phenomenon is that the network contains an unstable subsystem, which is stabilized by a feedback loop. The diffusion of molecules participating in this feedback loop then unleashes the inherent instability and allows growth of spatial modes. In the activator-inhibitor network in Figure 1, the activator plays the role of the unstable subsystem and the inhibitor provides the stabilizing feedback.

In this paper, we propose a new network, requiring only one diffusible component and an oscillator circuit serving as the unstable subsystem, as depicted in Figure 2. In Section II we cover the basic theory used in analyzing reactiondiffusion systems and relate Turing patterning to the concept of additive D-stability. In Section III we analyze a simplified theoretical model of our proposed architecture to demonstrate that it is indeed capable of generating patterns. In Section IV we propose a possible synthetic implementation that is built upon an available oscillator circuit using known genes and promoters, and provide an analysis of the system which predicts the range of real kinetic parameters over which patterns should emerge.

The primary goal of this paper is to provide a novel network architecture to serve as a starting point for exploring alternative implementation strategies for reaction-diffusion pattern generation by synthetic gene networks in multicellular systems. It thus focuses on preliminary analyses and not on experimental validation.

\section{REACTION-Diffusion System ANALYSis}

Turing pattern formation arises in reaction-diffusion systems where stability of a steady state in the reaction system does not imply stability of the homogeneous steady state in the presence of diffusion [12]. We will consider the situation where the cells are closely packed and study the continuous reaction-diffusion system

$$
\frac{\partial}{\partial t} c=f(c)+D \nabla^{2} c
$$




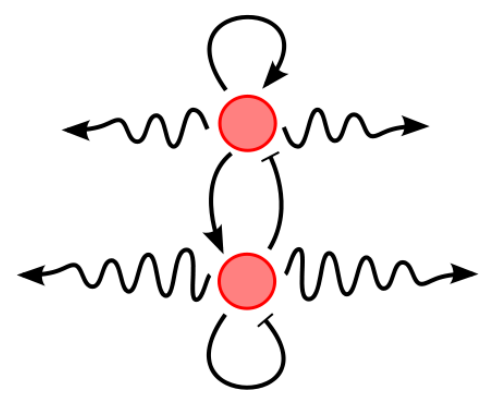

Fig. 1. The canonical two-component "activator-inhibitor" Turing system. The top component is the activator and the bottom component is the inhibitor, both diffusible.

over the spatial domain $\Omega$ with smooth boundary $\partial \Omega$ subject to zero-flux (Neumann) boundary conditions. Here $c(t, \xi)$ is the vector of species concentrations that depends on time $t$ and spatial variable $\xi \in \Omega, f$ is the vector field of reaction rates, $D \succeq 0$ is a diagonal matrix of diffusion coefficients, and $\nabla^{2}$ is the vector Laplacian. The Neumann boundary condition states that $\nabla c(t, \xi) \cdot n(\xi)=0, \forall \xi \in \partial \Omega$, where $n(\xi)$ is the outward normal vector.

We let $J=\left.\frac{\partial f}{\partial c}\right|_{c=c^{*}}$ denote the Jacobian linearization about the steady state $c^{*}$. The dynamical behavior of the reaction-diffusion system is determined from the matrices $J+\lambda_{k} D$, where $\lambda_{k}$ are the eigenvalues of the Laplacian operator $\nabla^{2}$ on the given spatial domain, and the subscripts $k=1,2,3, \ldots$ denote the wave numbers [18]. For example, on a one-dimensional domain $\Omega=[0, L], \lambda_{k}=-(\pi k / L)^{2}$. If the matrix $J+\lambda_{k} D$ is Hurwitz, then the corresponding wave decays to zero asymptotically in time. If $J+\lambda_{k} D$ is unstable, then the corresponding wave grows. In Turing's condition for pattern formation, matrix $J$ is stable, implying convergence to steady-state in the absence of diffusion, but $J+\lambda_{k} D$ is unstable for one or more wave numbers $k \geq 1$, implying the growth of these waves due to diffusion.

\section{A. Additive D-Stability}

A matrix stability concept that rules out Turing pattern formation is additive D-stability [19], [20], defined below. We are interested in this concept because necessary conditions for additive D-stability, when negated, serve as sufficient conditions for Turing instability.

Definition 1: A matrix $J$ is called additively D-stable if $J-\bar{D}$ is Hurwitz for all diagonal $\bar{D} \succeq 0$.

For Turing pattern formation, we need $J+\lambda_{k} D$ to become unstable for some $\lambda_{k}$. Because $\lambda_{k} \leq 0$ for Neumann eigenvalues [21] and all diffusion coefficients are non-negative, $J+\lambda_{k} D$ matches the $J-\bar{D}$ format of additive D-stability with $\bar{D}=-\lambda_{k} D$. Thus, if $J$ is additively D-stable, Turing pattern formation is not possible.

We will make use of the following necessary condition for additive D-stability and its proof to observe an essential structural property for Turing instability:

Theorem 1: A necessary condition for additive D-stability of the matrix $J$ is that $J$ and all of its principal submatrices be stable.

Proof: A result similar to Theorem 1 has been proven in [22]. Here, we present an alternative proof that makes explicit the structure of $\bar{D}$ that renders $J-\bar{D}$ unstable. Observing this structure will be helpful in designing a network that exhibits Turing instability.

We proceed by contradiction and suppose that $J \in \mathbb{R}^{n \times n}$ contains an unstable principal submatrix $J_{r}$ of size $r \leq n$, and show that we can recursively construct a diagonal matrix $\bar{D} \succeq 0$ such that $J-\bar{D}$ is unstable.

If $r=n$, then $J$ is an unstable matrix and we can choose $\bar{D}=0$. If $r<n$, we assume, without loss of generality, that $J_{r}$ is a leading principal submatrix. Taking the leading principal submatrix of size $r+1$ :

$$
J_{r+1}=\left[\begin{array}{cc}
J_{r} & b_{r} \\
c_{r} & a_{r+1}
\end{array}\right]
$$

we claim that we can find $\bar{D}_{r+1}=\operatorname{diag}\left\{0, \ldots, 0, d_{r+1}\right\}$ such that $J_{r+1}-\bar{D}_{r+1}$ is unstable. To see this, let $d_{r+1}=1 / \epsilon$ and note from standard singular perturbation arguments [23] that, as $\epsilon \rightarrow 0$, one of the eigenvalues of $J_{r+1}-\bar{D}_{r+1}$ approaches $-\infty$, while the remaining $r$ approach the eigenvalues of $J_{r}$. Since $J_{r}$ is unstable, then by an appropriately large choice of $d_{r+1}$ we can make $J_{r+1}-\bar{D}_{r+1}$ unstable.

Now we can similarly define

$$
J_{r+2}=\left[\begin{array}{cc}
J_{r+1} & b_{r+1} \\
c_{r+1} & a_{r+2}
\end{array}\right]
$$

and $\bar{D}_{r+2}=\operatorname{diag}\left\{0, \ldots, 0, d_{r+1}, d_{r+2}\right\}$, and render

$$
J_{r+2}-\bar{D}_{r+2}=\left[\begin{array}{cc}
J_{r+1}-\bar{D}_{r+1} & b_{r+1} \\
c_{r+1} & a_{r+2}-d_{r+2}
\end{array}\right]
$$

unstable by an appropriately large choice of $d_{r+2}$. We can then recursively apply this procedure until we make $J_{n}-\bar{D}_{n}=J-\bar{D}$ unstable using $\bar{D}=$ $\operatorname{diag}\left\{0, \ldots, 0, d_{r+1}, \ldots, d_{n}\right\}$.

Theorem 1 suggests that a possible route to Turing patterning is to break this necessary condition with an unstable principle submatrix. This means that an essential structural property for the Turing phenomenon is that the network contains an unstable subsystem. This subsystem must be stabilized by the rest of the system so that the matrix $J$ is stable, and the diffusion matrix $D$ must be such that $J+\lambda_{k} D$ is unstable for some wave number $k \geq 1$. A matrix $D$ with this property can indeed be constructed following the recursive procedure in the proof of Theorem 1.

\section{B. Activator-Inhibitor Example}

We show that the structural property for Turing instability discussed above encompasses the canonical two-component activator-inhibitor system in Figure 1. The linearization and diffusion matrices for this system have the form:

$$
J=\left[\begin{array}{ll}
j_{11} & j_{12} \\
j_{21} & j_{22}
\end{array}\right], \quad D=\left[\begin{array}{cc}
d_{1} & 0 \\
0 & d_{2}
\end{array}\right], \quad d_{i} \geq 0,
$$


where $j_{11}>0$ so that component 1 is the unstable "activator," and $j_{22}<0$ so that component 2 is the stabilizing "inhibitor." The activator thus serves as the unstable subsystem to disprove additive D-stability. The proof of Theorem 1 implies that instability is achieved when $d_{2}>0$ is large enough. The proof also implies that $d_{1}=0$ is admissible for Turing instability.

\section{QUENCHED OSCILLATOR SYSTEM}

We now present a new network architecture that is capable of generating Turing patterns. As discussed in the next section, this network can be implemented using existing synthetic biological components. It consists of a ring oscillator loop (represented with the pink molecules in Figure 2) that serves as the unstable subsystem and a second loop that "quenches" the oscillations and stabilizes the full system. The quenching loop contains a diffusible molecule (represented with blue in Figure 2), which means that when the corresponding diffusion coefficient is large, the diffusion matrix has the destabilizing structure proposed in the proof of Theorem 1.

For stability of the full system, it is essential that the quenching loop have a smaller phase lag than the oscillator loop. Smaller phase lag can be achieved with fewer reaction steps or with faster degradation rates in the second loop.

To demonstrate pattern formation, we consider the following representative model, which exhibits both an oscillator loop $\left(x_{1}, x_{2}, x_{3}\right)$ and a quenching loop $\left(x_{3}, x_{4}\right)$ :

$$
\begin{aligned}
\frac{\partial}{\partial t} x_{1} & =\frac{v_{1}}{1+x_{2}^{p}}-x_{1} \\
\frac{\partial}{\partial t} x_{2} & =\frac{v_{2}}{1+x_{3}^{p}}-x_{2} \\
\frac{\partial}{\partial t} x_{3} & =\frac{v_{3}}{1+x_{1}^{p}}+\frac{v_{4}}{1+x_{4}^{p}}-x_{3} \\
\frac{\partial}{\partial t} x_{4} & =\frac{v_{5} x_{3}^{p}}{1+\alpha x_{3}^{p}}-x_{4}+d_{4} \frac{\partial^{2} x_{4}}{\partial \xi^{2}}
\end{aligned}
$$

where the concentrations $x_{i}, i=1, \ldots, 4$, and all other variables and parameters are non-dimensional. In particular, the time variable $t$ is scaled to bring the degradation constants (assumed to be identical for each species) to one, and the one-dimensional length variable $\xi$ is scaled so that the spatial domain is $\Omega=[0, \pi]$. We assume only the fourth species (represented with blue in Figure 2) is diffusible and is subject to zero-flux boundary conditions. Because the fourth species is diffusible, this architecture is able to exhibit diffusiondriven instability for a large diffusion coefficent $d_{4}$.

Jacobian linearization of the reaction equations about the steady-state $\left(\bar{x}_{1}, \bar{x}_{2}, \bar{x}_{3}, \bar{x}_{4}\right)$ yields:

$$
J=\left[\begin{array}{ccc|c}
-1 & -b_{1} & 0 & 0 \\
0 & -1 & -b_{2} & 0 \\
-b_{3} & 0 & -1 & -c_{4} \\
\hline 0 & 0 & c_{5} & -1
\end{array}\right]
$$

where:

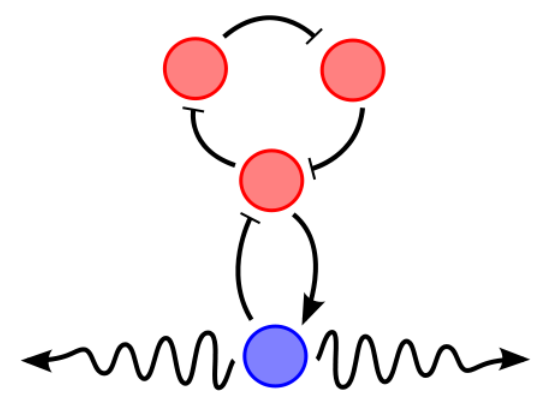

Fig. 2. The proposed "quenched oscillator" system. The quenching loop with the diffusible molecule (in blue) stabilizes the unstable oscillator loop (in pink). Diffusion then unleashes the inherent instability and allows growth of spatial modes with high wave numbers.

$$
\begin{gathered}
b_{1}=\frac{p v_{1} \bar{x}_{2}^{p-1}}{\left(1+\bar{x}_{2}^{p}\right)^{2}}, \quad b_{2}=\frac{p v_{2} \bar{x}_{3}^{p-1}}{\left(1+\bar{x}_{3}^{p}\right)^{2}}, \quad b_{3}=\frac{p v_{3} \bar{x}_{1}^{p-1}}{\left(1+\bar{x}_{1}^{p}\right)^{2}}, \\
c_{4}=\frac{p v_{4} \bar{x}_{4}^{p-1}}{\left(1+\bar{x}_{4}^{p}\right)^{2}}, \quad c_{5}=\frac{p v_{5} \bar{x}_{3}^{p-1}}{\left(1+\alpha \bar{x}_{3}^{p}\right)^{2}} .
\end{gathered}
$$

For the oscillator subsystem, we require:

$$
B \triangleq b_{1} b_{2} b_{3}>8
$$

so that the characteristic polynomial of the $3 \times 3$ upper-left principal submatrix of $J$, given by $(\lambda+1)^{3}+B$, has a pair of complex conjugate roots in the right half-plane.

For stability of the full reaction network, we need:

$$
C \triangleq c_{4} c_{5}>\frac{B-8}{2}
$$

so that $\operatorname{det}(\lambda I-J)=(\lambda+1)\left[(\lambda+1)^{3}+B+C(\lambda+1)\right]$ has all roots in the left half-plane. For diffusion-driven instability of the $k^{t h}$ spatial mode $\cos (k \xi)$, the polynomial:

$$
\begin{aligned}
\operatorname{det}( & \left.\lambda I-\left(J-k^{2} \operatorname{diag}\left\{0,0,0, d_{4}\right\}\right)\right)= \\
& (\lambda+1)\left[(\lambda+1)^{3}+B+C(\lambda+1)\right] \\
& +k^{2} d_{4}\left[(\lambda+1)^{3}+B\right]
\end{aligned}
$$

where $d_{4}$ is the diffusion coefficient, must have right halfplane roots. Indeed, when the product $k^{2} d_{4}$ is sufficiently large, three roots of (6) approach those of $(\lambda+1)^{3}+B$, which contain right-half plane roots due to (4). This means that the inhomogeneous modes $\cos (k \xi)$ grow in time if $k^{2} d_{4}$ exceeds the threshold for instability of the polynomial (6).

The parameters $p=3, v_{1}=v_{2}=18, v_{3}=v_{4}=0.45$, and $\alpha=0.1$ in the system (2) satisfy conditions (4)-(5) with $B=9.4815, C=2.2222$, and the polynomial (6) becomes unstable when $k^{2} d_{4}>11.35$. Simulations in Figure $3 \mathrm{a}$ indeed exhibit growth of the spatial inhomogeneity when the steady state is perturbed by adding the first wave $(k=1)$ with a small amplitude. This Turing behavior is contrasted in Figure $3 b$ to the decay of the initial inhomogeneity in the absence of diffusion.

\section{IMPLEMENTATION OF THE QUENCHED OSCILLATOR}

A possible synthetic implementation of the quenched oscillator system is depicted in Figure 4. The first (top) loop is the repressilator [24], which is a ring oscillator, 

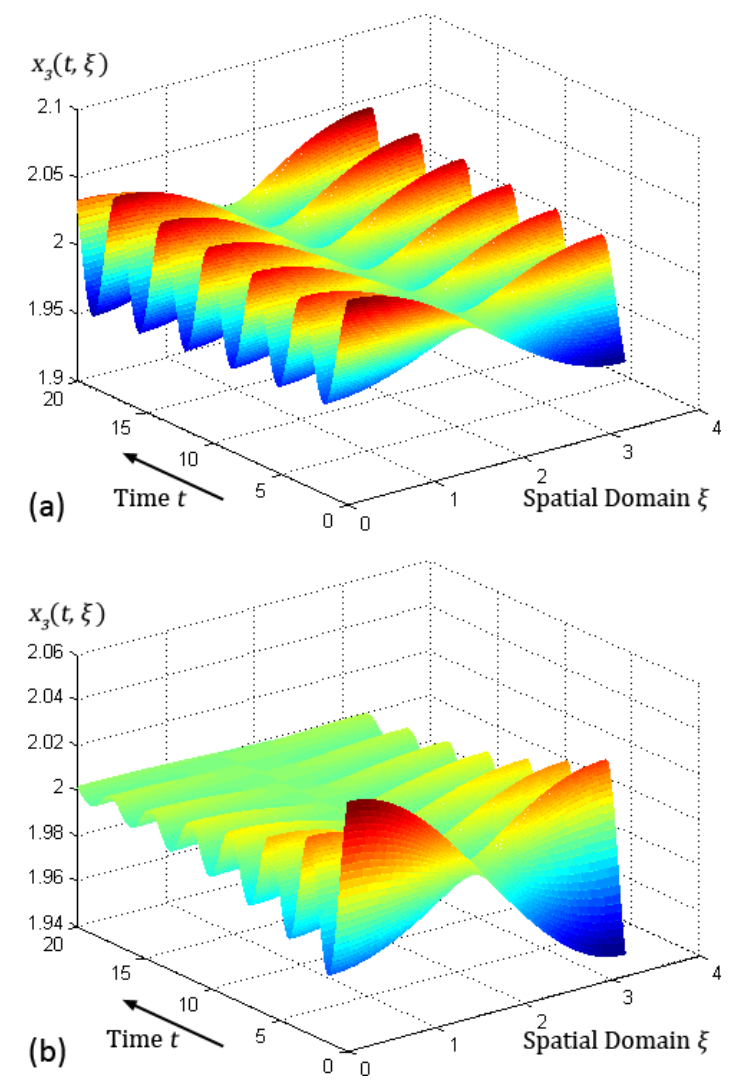

Fig. 3. (a) Solution $x_{3}(t, \xi)$ of (2) on spatial domain $\Omega=[0, \pi]$ with zero-flux boundary condition. With parameters $p=3, v_{1}=v_{2}=18$, $v_{3}=v_{4}=9, v_{5}=0.45, \alpha=0.1, d_{4}=20$, Turing instability conditions are met and a slight perturbation of the homogenous initial condition with the first wave $(k=1)$ leads to growth of the inhomogeneity. (b) Simulations in part (a) repeated with the diffusion coefficient $d_{4}$ set to zero. The initial homogeneity decays in time.

comprised of three pairs of transcriptional repressors (tetR, lacI, $\lambda(c I)$ and promoters $\left(P_{\text {Ltet } O-1}, P_{\text {LlacO-1 }}, \lambda P_{R}\right)$. The second (bottom) loop has one inhibitory interaction and includes the membrane-diffusible signaling molecule acylehomoserine lactone $(A H L)$ as well as $V$. fischeri quorum sensing genes luxI and luxR. The second loop interacts with the first loop through tetR and in a single system will cause the first loop to cease oscillations.

Although the oscillations produced by the repressilator are known to be noisy and irregular in practice, for our application of pattern generation, the interest is not in the quality of oscillations, but in the presence of unstable modes. The repressilator was chosen because it is a seminal example of a synthetic oscillator and also matches the oscillator design based on a phase lag mechanism as in Figure 2.

\section{A. System PDE Model}

We represent the dynamics of this network with the following set of partial differential equations:

$$
\begin{aligned}
\frac{\partial}{\partial t} m_{C}= & V_{P_{L t e t O-1}} N_{C} C\left(\frac{1}{1+\left(\frac{p_{T}}{K_{T}}\right)^{n_{T}}}+\ell_{P_{\text {LtetO-1 }}}\right) \\
& -\gamma_{m O} m_{C}
\end{aligned}
$$

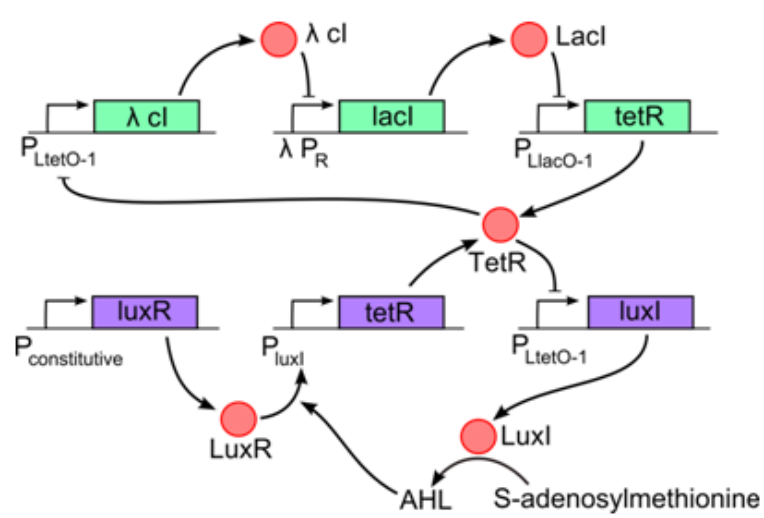

Fig. 4. A possible synthetic implementation of the network in Figure 2. Two feedback loops are interconnected by shared production and sensing of the transcriptional repressor tetR. The genes in the first feedback loop are depicted in green and the genes in the second feedback loop are shown in purple. The second loop contains the membrane-diffusable signaling molecule acyle-homoserine lactone $(A H L)$.

$$
\begin{aligned}
\frac{\partial}{\partial t} p_{C}= & \epsilon_{C} m_{C}-\gamma_{C} p_{C} \\
\frac{\partial}{\partial t} m_{T O}= & V_{P_{\text {Llac } O-1}} N_{T O} C\left(\frac{1}{1+\left(\frac{p_{L}}{K_{L}}\right)^{n_{L}}}+\ell_{P_{\text {LlacO-1 }}}\right) \\
\frac{\partial}{\partial t} p_{T}= & \epsilon_{\text {mOO }} m_{T O} m_{T O}+\epsilon_{T Q} m_{T Q}-\gamma_{T} p_{T} \\
\frac{\partial}{\partial t} m_{L}= & V_{P_{R}} N_{L} C\left(\frac{1}{1+\left(\frac{p_{C}}{K_{C}}\right)^{n_{C}}}+\ell_{P_{R}}\right) \\
& -\gamma_{m O} m_{L} \\
\frac{\partial}{\partial t} p_{L}= & \epsilon_{L} m_{L}-\gamma_{L} p_{L} \\
\frac{\partial}{\partial t} m_{I}= & V_{P_{L t e t O-1}} N_{I} C\left(\frac{1}{1+\left(\frac{p_{T}}{K_{T}}\right)^{n_{T}}}+\ell_{P_{L t e t O-1}}\right) \\
& -\gamma_{m Q} m_{I} \\
\frac{\partial}{\partial t} p_{I}= & \epsilon_{I} m_{I}-\gamma_{I} p_{I} \\
\frac{\partial}{\partial t} A= & v_{3} p_{I}-k_{f} A\left(p_{R}-p_{R A}\right)+k_{r} p_{R A}-\gamma_{A} A \\
& +d_{A H L} \nabla^{2} A \\
\frac{\partial}{\partial t} p_{R A}= & k_{f} A\left(p_{R}-p_{R A}\right)-k_{r} p_{R A} \\
\frac{\partial}{\partial t} m_{T Q}= & V_{P_{L u x I}} N_{T Q} C\left(\frac{1}{1+\left(\frac{K_{R A}}{p_{R A}}\right)^{n_{R A}}}+\ell_{P_{L u x I}}\right) \\
& -\gamma_{m Q} m_{T Q}
\end{aligned}
$$

where $V_{i}$ are velocity constants, $N_{i}$ are copy numbers, $K_{i}$ are dissociation constants, $n_{i}$ are Hill coefficients, $\ell_{i}$ are leakage rates normalized to $V_{i}, \gamma_{i}$ are degradation rates, and $\epsilon_{i}$ are protein translational rates. The parameters are subscripted according to their corresponding species $(C=[c I]$, $T=[$ tet $R], L=[$ lacl $], I=[$ luxl] $, A=[A H L], R=[\operatorname{luxR}], R A=[$ luxR $A H L$ complex]) except for velocity and leakage constants, which are subscripted by promoter, and copy numbers, which are subscripted by the gene being transcribed. The concentration of the mRNA for tetR is split into those produced by the oscillator loop $(O)$ and the quenching loop $(Q)$. The 
parameter $C$ is the concentration level generated by a single molecule in our spatial domain and $d_{A H L}$ is the diffusion coefficient of AHL. We take $\gamma_{C}=\gamma_{T}=\gamma_{L} \triangleq \gamma_{p}$.

\section{B. Conditions for Patterning}

The linearization of the reaction terms in (7)-(17) at steady state $\left(\bar{m}_{C}, \bar{p}_{C}, \bar{m}_{T O}, \bar{p}_{T}, \bar{m}_{L}, \bar{p}_{L}, \bar{m}_{I}, \bar{p}_{I}, \bar{A}, \bar{p}_{R A}, \bar{m}_{T Q}\right)$ yields the Jacobian matrix:

$$
J=\left[\begin{array}{l|l}
J_{11} & J_{12} \\
\hline J_{21} & J_{22}
\end{array}\right]
$$

with the submatrices:

$$
\begin{aligned}
& J_{11}=\left[\begin{array}{cccccc}
-\gamma_{m O} & 0 & 0 & -b_{4} & 0 & 0 \\
\epsilon_{C} & -\gamma_{C} & 0 & 0 & 0 & 0 \\
0 & 0 & -\gamma_{m O} & 0 & 0 & -b_{6} \\
0 & 0 & \epsilon_{T O} & -\gamma_{T} & 0 & 0 \\
0 & -b_{2} & 0 & 0 & -\gamma_{m O} & 0 \\
0 & 0 & 0 & 0 & \epsilon_{L} & -\gamma_{L}
\end{array}\right] \\
& J_{12}=\left[\begin{array}{ccccc}
0 & 0 & 0 & 0 & 0 \\
0 & 0 & 0 & 0 & 0 \\
0 & 0 & 0 & 0 & 0 \\
0 & 0 & 0 & 0 & \epsilon_{T Q} \\
0 & 0 & 0 & 0 & 0 \\
0 & 0 & 0 & 0 & 0
\end{array}\right] \\
& J_{21}=\left[\begin{array}{cccccc}
0 & 0 & 0 & -b_{42} & 0 & 0 \\
0 & 0 & 0 & 0 & 0 & 0 \\
0 & 0 & 0 & 0 & 0 & 0 \\
0 & 0 & 0 & 0 & 0 & 0 \\
0 & 0 & 0 & 0 & 0 & 0
\end{array}\right] \\
& J_{22}=\left[\begin{array}{ccccc}
-\gamma_{m Q} & 0 & 0 & 0 & 0 \\
\epsilon_{I} & -\gamma_{I} & 0 & 0 & 0 \\
0 & v_{3} & -a_{9} & a_{10} & 0 \\
0 & 0 & c_{9} & -a_{10} & 0 \\
0 & 0 & 0 & b_{10} & -\gamma_{m Q}
\end{array}\right]
\end{aligned}
$$

where we use the parameters $\alpha_{C} \triangleq \bar{p}_{C} / K_{C}, \alpha_{T} \triangleq \bar{p}_{T} / K_{T}$, $\alpha_{L} \triangleq \bar{p}_{L} / K_{L}$, and $\alpha_{A} \triangleq \bar{A} / K_{A}$ to obtain the off-diagonal entries:

$$
\begin{aligned}
c_{9} & =\frac{k_{f} p_{R}}{1+\alpha_{A}}, \\
a_{9} & =c_{9}+\gamma_{A}, \\
a_{10} & =k_{r}\left(1+\alpha_{A}\right) \\
b_{2} & =V_{P_{R}} V_{L} C \frac{n_{C} \alpha_{C}^{\left(n_{C}-1\right)}}{K_{C}\left(1+\alpha_{C}^{n_{C}}\right)^{2}}, \\
b_{4} & =V_{P_{L t e t O-1}} N_{C} C \frac{n_{T} \alpha_{T}^{\left(n_{T}-1\right)}}{K_{T}\left(1+\alpha_{T}^{n_{T}}\right)^{2}}, \\
b_{42} & =V_{P_{\text {LtetO-1 }}} N_{I} C \frac{n_{T} \alpha_{T}^{\left(n_{T}-1\right)}}{K_{T}\left(1+\alpha_{T}^{n_{T}}\right)^{2}}, \\
b_{6} & =V_{P_{\text {LlacO-1 }}} N_{T O} C \frac{\left.n_{L} \alpha_{L}^{(n}-1\right)}{K_{L}\left(1+\alpha_{L}^{n_{L}}\right)^{2}}, \\
b_{10} & =V_{P_{L u x I}} N_{T Q} C \frac{n_{R A}\left(\frac{K_{R A}}{p_{R}}\right)\left(\frac{K_{R A}}{p_{R}} \frac{1+\alpha_{A}}{\alpha_{A}}\right)^{n_{R A}}}{p_{R}\left(1+\left(\frac{K_{R A}}{p_{R}} \frac{1+\alpha_{A}}{\alpha_{A}}\right)^{n_{R A}}\right)^{2}} .
\end{aligned}
$$

We note that $J_{11}$ is the linearization matrix for the first loop, which corresponds to the standard repressilator system ( $c I$ lacI-tetR).

The eigenvalues of $J_{11}$ are the roots of:

$\operatorname{det}\left(s I-J_{11}\right)=\left(s+\gamma_{m O}\right)^{3}\left(s+\gamma_{p}\right)^{3}+\epsilon_{C} \epsilon_{T O} \epsilon_{L} b_{2} b_{4} b_{6}$.

It can be shown [24] that instability of $J_{11}$ is achieved when:

$$
\frac{(\beta+1)^{2}}{\beta}<\frac{3 X^{2}}{4+2 X}
$$

where $\beta \triangleq \gamma_{p} / \gamma_{m O}$ and $X \triangleq-\frac{1}{\gamma_{p} \gamma_{m O}} \sqrt[3]{\epsilon_{C} \epsilon_{T O} \epsilon_{L} b_{2} b_{4} b_{6}}$. Substituting and rearranging, we arrive at the following expression for $X$ :

$$
\begin{aligned}
X^{3}= & -n_{C} n_{T} n_{L} \frac{\alpha_{R A}}{1+\alpha_{R A}} \frac{\alpha_{C}^{n_{C}}}{1+\alpha_{C}^{n_{C}}} \frac{1}{1+\ell_{P_{R}}\left(1+\alpha_{C}^{n_{C}}\right)} \\
& \times \frac{\alpha_{T}^{n_{T}}}{1+\alpha_{T}^{n_{T}}} \frac{1}{1+\ell_{P_{L t e t O-1}}\left(1+\alpha_{T}^{n_{T}}\right)} \\
& \times \frac{\alpha_{L}^{n_{L}}}{1+\alpha_{L}^{n_{L}}} \frac{1}{1+\ell_{P_{L l a c O-1}}\left(1+\alpha_{L}^{n_{L}}\right)},
\end{aligned}
$$

where the additional variable $\alpha_{R A} \geq 0$ is defined by the relation:

$\frac{1}{1+\alpha_{R A}} \triangleq \frac{\epsilon_{T Q} V_{P_{L u x I}} N_{T Q} C}{\gamma_{p} \gamma_{m Q} \alpha_{T} K_{T}}\left(\frac{1}{1+\left(\frac{K_{R A}}{p_{R}} \frac{1+\alpha_{A}}{\alpha_{A}}\right)^{n_{R A}}}+\ell_{P_{L u x I}}\right)$.

The eigenvalues of $J$ are the roots of:

$$
\begin{aligned}
\operatorname{det}(s I-J)= & \operatorname{det}\left(s I-J_{11}\right)\left(s+\gamma_{I}\right)\left(s+\gamma_{m Q}\right)^{2} \\
& \times\left[\left(s+a_{9}\right)\left(s+a_{10}\right)-c_{9} a_{10}\right] \\
& +F\left(s+\gamma_{m O}\right)^{3}\left(s+\gamma_{p}\right)^{2}
\end{aligned}
$$

where $F \triangleq v_{3} \epsilon_{I} \epsilon_{T Q} c_{9} b_{42} b_{10}$ characterizes the feedback strength. $F$ must be a value such that all of the eigenvalues of $J$ are stable. Substituting and rearranging, we arrive at the following expression for $F$ :

$$
\begin{aligned}
F= & \gamma_{T} \gamma_{I} \gamma_{A} \gamma_{m Q}^{2} k_{r} n_{T} n_{R A} \frac{1}{1+\alpha_{R A}} \frac{\left(\frac{K_{R A}}{p_{R}} \frac{1+\alpha_{A}}{\alpha_{A}}\right)^{n_{R A}}}{1+\left(\frac{K_{R A}}{p_{R}} \frac{1+\alpha_{A}}{\alpha_{A}}\right)^{n_{R A}}} \\
& \times \frac{1}{1+\ell_{P_{L u x I}}\left(1+\left(\frac{K_{R A}}{p_{R}} \frac{1+\alpha_{A}}{\alpha_{A}}\right)^{n_{R A}}\right)} \\
& \times \frac{\alpha_{T}^{n_{T}}}{1+\alpha_{T}^{n_{T}}} \frac{1}{1+\ell_{P_{\text {LtetO }-1}}\left(1+\alpha_{T}^{n_{T}}\right)}
\end{aligned}
$$

Here $D=\operatorname{diag}\left\{0,0,0,0,0,0,0,0, d_{A H L}, 0,0\right\} . J+\lambda_{k} D$ looks identical to $J$ except for the $A H L$ entry of the diagonal, which is now defined as $-\hat{a}_{9} \triangleq-c_{9}-\gamma_{A}+\lambda_{k} d_{A H L}$. This leads to: 


$$
\begin{aligned}
\operatorname{det}\left(s I-\left(J+\lambda_{k} D\right)\right)= & \operatorname{det}\left(s I-J_{11}\right)\left(s+\gamma_{I}\right)\left(s+\gamma_{m Q}\right)^{2} \\
& \times\left[\left(s+\hat{a}_{9}\right)\left(s+a_{10}\right)-c_{9} a_{10}\right] \\
& +F\left(s+\gamma_{m O}\right)^{3}\left(s+\gamma_{p}\right)^{2},
\end{aligned}
$$

which yields unstable roots for large enough $\lambda_{k} d_{A H L}$. This means that for diffusion-driven patterning we need a large diffusion coefficient, a large wave number, or a small spatial domain.

\section{Parameter Selection}

This system was constructed using relatively well-studied components. Some parameter values have been experimentally measured, and accepted literature values were used whenever possible. Other parameters were estimated based on values of similar molecules or within a known range of manipulation. For example, protein degradation rates can be controlled by adding or changing the ssrA tag [25].

Taking some parameters to be fixed, we use the above results to guide our choices for manipulatable values by checking that the first two conditions for Turing patterning are met and that experimentally-reasonable wave numbers become unstable. We reduce the number of parameters in the system by treating the steady state values $\alpha_{C}, \alpha_{T}, \alpha_{L}$, $\alpha_{A}$, and $\alpha_{R A}$ as new parameters to be adjusted such that $|X|$ is large enough for instability of $J_{11}$ and $F$ is large enough to stabilize the overall system, then solve for the original set of parameters. For example, $\alpha_{C}^{*}={ }^{2 n} \sqrt{1+1 / \ell_{P_{R}}}$ will maximize $|X|$ for that variable and increasing $k_{r}$ and $k_{f}$ proportionally will increase $F$ without affecting the steadystate values.

Following this procedure, we generated a list of plausible parameter values that meet the conditions for Turing patterning, which is available upon request.

\section{CONClusions AND Future WORK}

We have proposed here a novel network architecture for reaction-diffusion pattern generation in gene expression that we call a quenched oscillator network. We presented both a simplified theoretical model and a possible synthetic implementation to demonstrate this network's ability to produce patterns. The eventual goal is a laboratory implementation that exhibits Turing patterning. According to our parameter list, most of the steady state concentrations are on the order of $10^{-9}$, which means stochastic effects will be significant. Stochastic simulations [26] will allow us to better evaluate the experimental plausibility of the proposed system. Future directions include trying different, possibly more robust, oscillators [27] or different diffusible components.

\section{REFERENCES}

[1] S. Kondo and R. Asai, "A reaction-diffusion wave on the skin of the marine angelfish Pomacanthus," Nature, vol. 376, no. 6543, pp. 765768, 1995.

[2] M. Yamaguchi, E. Yoshimoto, and S. Kondo, "Pattern regulation in the stripe of zebrafish suggests an underlying dynamic and autonomous mechanism," Proceedings of the National Academy of Sciences, vol. 104 , no. 12, p. 4790, 2007.
[3] H. Levine and W. Rappel, "Membrane-bound Turing patterns," Physical Review E, vol. 72, no. 6, p. 61912, 2005.

[4] D. Strier and S. Dawson, "Role of complexing agents in the appearance of Turing patterns," Physical Review E, vol. 69, no. 6, p. 66207, 2004

[5] Q. Ouyang and H. Swinney, "Transition from a uniform state to hexagonal and striped Turing patterns," Nature, vol. 352, no. 6336, pp. 610-612, 1991

[6] J. Horvath, I. Szalai, and P. De Kepper, "An experimental design method leading to chemical Turing patterns," Science, vol. 324, no. 5928, p. 772, 2009.

[7] R. Dillon, P. Maini, and H. Othmer, "Pattern formation in generalized Turing systems," Journal of Mathematical Biology, vol. 32, no. 4, pp. 345-393, 1994

[8] M. Alber, T. Glimm, H. Hentschel, B. Kazmierczak, and S. Newman, "Stability of n-dimensional patterns in a generalized Turing system: implications for biological pattern formation," Nonlinearity, vol. 18 p. $125,2005$.

[9] V. Castets, E. Dulos, J. Boissonade, and P. De Kepper, "Experimental evidence of a sustained standing Turing-type nonequilibrium chemical pattern," Physical Review Letters, vol. 64, no. 24, pp. 2953-2956, 1990.

[10] Q. Ouyang, Z. Noszticzius, and H. Swinney, "Spatial bistability of two-dimensional Turing patterns in a reaction-diffusion system," The Journal of Physical Chemistry, vol. 96, no. 16, pp. 6773-6776, 1992

[11] I. Szalai and P. De Kepper, "Turing Patterns, Spatial Bistability, and Front Instabilities in a Reaction- Diffusion System," J. Phys. Chem. A, vol. 108, no. 25, pp. 5315-5321, 2004.

[12] A. Turing, "The Chemical Basis of Morphogenesis," Philosophical Transactions, Royal Society of London B-237: 32, vol. 72, pp. 37-72, 1952.

[13] D. Cohen, R. Morfino, and M. Maharbiz, "A Modified Consumer Inkjet for Spatiotemporal Control of Gene Expression," PLoS ONE, vol. 4, p. e7086, 2009.

[14] T. Sohka, R. Heins, and M. Ostermeier, "Morphogen-defined patterning of Escherichia coli enabled by an externally tunable band-pass filter," Journal of biological engineering, vol. 3, no. 1, p. 10, 2009.

[15] S. Basu, Y. Gerchman, C. Collins, F. Arnold, and R. Weiss, "A synthetic multicellular system for programmed pattern formation," Nature, vol. 434, no. 7037, pp. 1130-1134, 2005.

[16] S. Basu, R. Mehreja, S. Thiberge, M. Chen, and R. Weiss, "Spatiotemporal control of gene expression with pulse-generating networks," Proceedings of the National Academy of Sciences of the United States of America, vol. 101, no. 17, p. 6355, 2004.

[17] E. Lucchetta, J. Lee, L. Fu, N. Patel, and R. Ismagilov, "Dynamics of Drosophila embryonic patterning network perturbed in space and time using microfluidics," Nature, vol. 434, no. 7037, pp. 1134-1138, 2005.

[18] R. Casten and C. Holland, "Stability properties of solutions to systems of reaction-diffusion equations," SIAM Journal on Applied Mathematics, pp. 353-364, 1977.

[19] X. Ge and M. Arcak, "A sufficient condition for additive D-stability and application to reaction-diffusion models," Systems \& Control Letters, vol. 58, no. 10-11, pp. 736-741, 2009.

[20] E. Kaszkurewicz and A. Bhaya, Matrix diagonal stability in systems and computation. Birkhauser, 2000.

[21] H. Gottlieb, "Eigenvalues of the Laplacian with Neumann boundary conditions," The Journal of the Australian Mathematical Society. Series B. Applied Mathematics, vol. 26, pp. 293-309, 1985.

[22] H. Othmer, "Synchronized and differentiated modes of cellular dynamics," Dynamics of Synergetic Systems. Berlin Heidelberg New York: Springer, 1980.

[23] P. Kokotović, H. Khalil, and J. O'Reilly, Singular Perturbation Methods in Control: Analysis and Design. Academic Press, 1986.

[24] M. Elowitz and S. Leibler, "A synthetic oscillatory network of transcriptional regulators," Nature, vol. 403 , no. 6767 , pp. 335-338, 2000

[25] E. Jabri, "Tag, you're degraded," Nature Structural \& Molecular Biology, vol. 10, no. 9, p. 676, 2003.

[26] M. Lis, M. Artyomov, S. Devadas, and A. Chakraborty, "Efficient stochastic simulation of reaction-diffusion processes via direct compilation," Bioinformatics, vol. 25, no. 17, pp. 2289-2291, 2009.

[27] J. Stricker, S. Cookson, M. Bennett, W. Mather, L. Tsimring, and J. Hasty, "A fast, robust and tunable synthetic gene oscillator," Nature, vol. 456, no. 7221, pp. 516-519, 2008. 